\title{
Ultrasound-guided fluoroscopic-verified trans-foraminal lumbar dorsal root ganglion pulsed radiofrequency modulation for radicular pain relief: clinical and cadaveric evaluation of the technique
}

\author{
Shaaban Omar ${ }^{1 *}$, Sherif El-Sayed Hegab', Mohamed Ihab Samy Reda', Sahar Ahmed El-Karadawy², \\ Mowaffak Moustafa Saad ${ }^{3}$, Nancy Mohamed El Sekily ${ }^{4}$ and Omar Sameh Elaassar ${ }^{1}$
}

\begin{abstract}
Background: Ultrasound-guided lumbar pain interventions were thought to be difficult; the high acoustic impedance of bone hides the underling structures and needle path. Reviewing the sonoanatomy of the lumbar region using different planes and angles made better sonographic guidance for spine injections. The aim of this prospective study is to assess the accuracy and safety of ultrasound (US)-guided lumbar trans-foraminal pulsed radiofrequency of the dorsal root ganglion confirmed by fluoroscopic imaging in management of chronic radicular pain.

Results: Thirty-two patients, with 34 lumbar interventions, were included in the study. Thirty-one interventions out of 34 were performed successfully with overall accuracy of $91.18 \%$ and with minimal complications. The successful first trial placement of the cannula was calculated in $44.1 \%$ of interventions; multiple trials were needed in $47.1 \%$ while incorrect level was encountered in 8.8\%. Visual analogue scale of pain and Oswestry Disability Index decreased significantly after intervention up to 3 months compared to the pre-intervention value. The analgesic consumption was reduced by mean of $73.44 \pm 31.07 \% 1$ month after intervention.
\end{abstract}

Conclusions: US-guided fluoroscopic-verified trans-foraminal PR of lumbar DRG is accurate, safe, and effective for CRP.

Keywords: Pulsed radiofrequency, Lumbar DRG, Ultrasound, Fluoroscopy

\section{Background}

Lumbar radicular pain is defined as a pain that radiates from back into the lower extremity along the course of a spinal nerve root [1]. The most common cause of radicular pain is disc herniation that is diagnosed clinically and radiologically using MRI [2]. Conservative treatment (pharmacotherapy or physiotherapy) is effective management in $2 / 3$ of patients, yet pain continues to be chronic

\footnotetext{
* Correspondence: shaaban.radiology@gmail.com

'Department of Diagnostic and Interventional Radiology, Faculty of

Medicine, Alexandria University, Alexandria, Egypt

Full list of author information is available at the end of the article
}

in the remaining cases and affects patient physically and psychologically [3].

Many minimally invasive interventions are tried for chronic radicular pain due to disc herniation, including nerve root or epidural injection, endoscopic discectomy, spinal cord, or dorsal root ganglion (DRG) stimulation and modulation [4].

DRG ablation or stimulation is a novel therapeutic option for chronic radicular pain [4]. DRGs are 31 pairs and convey all sensory information including pain from the periphery to the spinal cord. Mechanical compression or irritation to DRGs liberates pain mediators and 
initiates ectopic firing that once transmitted to spinal cord induces central sensitization; therefore, DRGs have become an important target area for treating radicular pain [5].

DRGs have different locations and sizes along the vertebral column. In the lumbar region, the sizes of the DRGs increase progressively from L1 to a maximum at L5, and the distribution is either foraminal or extra foraminal. Morphologically, DRG may be singular, biganglia, or triganglia [6].

Pulsed radiofrequency (PRF) is the technique commonly used for neural modulation of DRGs. PRF generates a radiofrequency current in short, high-voltage bursts with intermission for enough heat dissipation time to ensure temperature not higher than $42^{\circ} \mathrm{C}$. Thus, it suppresses the action potential transmission by neurons with no neural damage [7].

Fluoroscopic guidance is an imaging guide that is strongly recommended in interventional spine procedures including PRF modulation of DRGs. Radiation exposure is the major health hazard associated with performing fluoroscopic images [8].

Growing interest of ultrasound (US)-guided intervention techniques in chronic pain encourages its use in the spine region [9]. The advantages of using ultrasound over other radiological modalities are no ionizing radiation hazards, better visualization of soft tissues (i.e., muscle, ligament) and blood vessels, and a real-time visualization of needle advancement. It is portable and is cost-effective. However, several concerns such as accuracy and precision, reliability, and patient safety should be addressed [10].

The current study investigated the accuracy and safety of US-guided trans-foraminal lumbar dorsal root ganglion in management of chronic radicular pain (CRP) with subsequent fluoroscopy (FL) confirmation.

\section{Methods}

The current prospective descriptive study was done by two operators, a consultant of pain management and radiologist, and conducted primarily on one preserved cadaver then continued on 32 adult patients. The sample size was calculated for agreement study of US for the radiological guidance of trans-foraminal needle placement targeting the lumbar dorsal root ganglion using PRF modulation to relieve patients' radicular pain [11].

The study protocol was approved by the Ethical Review Board and a written informed consent was obtained from each patient.

All patients fulfilled the inclusion criteria; they all had lumbar CRP for more than 3 months due to a localized herniated disc shown in MRI, not responding and/or intolerant to conservative treatment (visual analogue scale
(VAS) of pain measuring more than 4), and reporting no progressive motor weakness.

Patients were excluded if they reported a history of allergy to local anesthetics or radiographic contrast medium, spinal deformity or previous lumbar spinal surgery, psychiatric disorders, local or systemic contraindication to lumbar interventions, and pregnancy.

The primary outcome of the present study was to measure the grade of accuracy of US-guided transforaminal PR cannula placement adjacent to the desired DRG; secondary outcomes were pain relief, analgesic consumption, patients' functionality, and safety of the technique.

\section{The intervention technique}

After reviewing Provenzano and Narouze's study [12] which described 7 sonographic views of lumbar spine injections and the studies published by Gofeld et al. [13] and Galiano et al. [14] which described US-guided 1umbar trans-foraminal injection, the current study suggested that axial sonogram of lumbar vertebra below the level of desired transverse process, targeting lateral border of inferior articular process using in-plane needle approach, is the best plane for trans-foraminal DRG modulation.

\section{First stage of the study (cadaveric work)}

A preserved whole-body cadaver was placed in prone position on a procedural table at the anatomy laboratory. Personal protective tools were worn and a curved US probe $(25 \mathrm{MHz})$ of US GE machine (logic P5, USA) was covered and placed midline sagittal oblique to view the sacral hiatus, sacrum and lumbar spinous processes, and laminae.

At the selected vertebral level, the US probe was rotated transversely to view the axial sonogram of the spinous process, superior articular process, lamina, and transverse process (TP) (Fig. 1). The US probe was moved slightly down and/or oblique below the level of TP with mild cephalic tilt to visualize inferior edge of lamina, lateral border of inferior articular process, dura, and existing nerve root (Fig. 2). One-finger breadth lateral to the lateral edge of the probe a spinal needle was inserted approximately $70-80^{\circ}$ on the cadaver skin and directed from lateral to medial towards the lateral border of the inferior articular process (Fig. 3).

Upon reaching the lateral border of the articular process, the angle of the needle on the cadaver surface decreased (to $60^{\circ}$ and less) during the needle advancement anterior, superior. and medial to the articular process to be placed into the intervertebral foramen; then, $1 \mathrm{cc}$ of ionized contrast was injected and only an antero-posterior (A-P) fluoroscopic view was taken to verify the vertebral level (Fig. 4) and the dye spread 


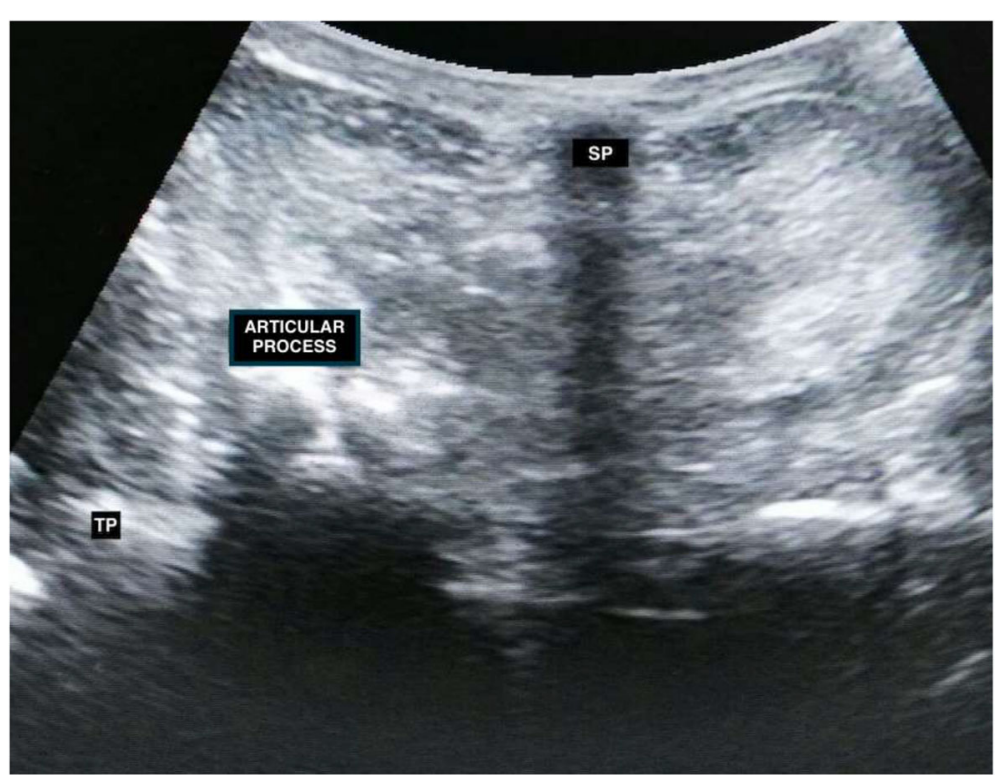

Fig. 1 Axial inter laminar sonogram of cadaveric lumbar vertebra shows shadow of spinous process (SP), transverse process (TP), articular process $(\mathrm{AP})$, and dura

pattern in the dural sleeve around the nerve root (Fig. 5) FL machine was designed for A-P view only. Five trans-foraminal injections (L1/L2 through L5/S1) were performed bilaterally.

The aim of this stage was to make the best needle probe orientation; determination of the site, direction, tilt, and angle of needle entry; and path into the transforaminal space under US guidance.

\section{Second stage of the study (clinical work)}

The intervention was done in the block room under complete aseptic technique. The patient asked to be in prone position on the operating table with a pillow under his/her abdomen to eliminate lumbar lordosis. A portable US machine (SonoSite Inc., US) with a curved probe $(2-5 \mathrm{MHz}$ frequency) was adjusted and used for vertebral counting to reach the desired vertebral level simulating the technique performed on the cadaver (Figs. 6, 7, 8, and 9).

Fluoroscopic imaging was taken for level verification by fluoroscopy machine, Siemens, Cios Connect. Two milliliters of lidocaine $1 \%$ was injected to numb the skin and underlying tissues and a 22-G, 90-mm PRF insulated /curved cannula, with a $10-\mathrm{mm}$ active tip, was inserted into the desired intervertebral foramen using the abovementioned cadaveric technique (Fig. 10).
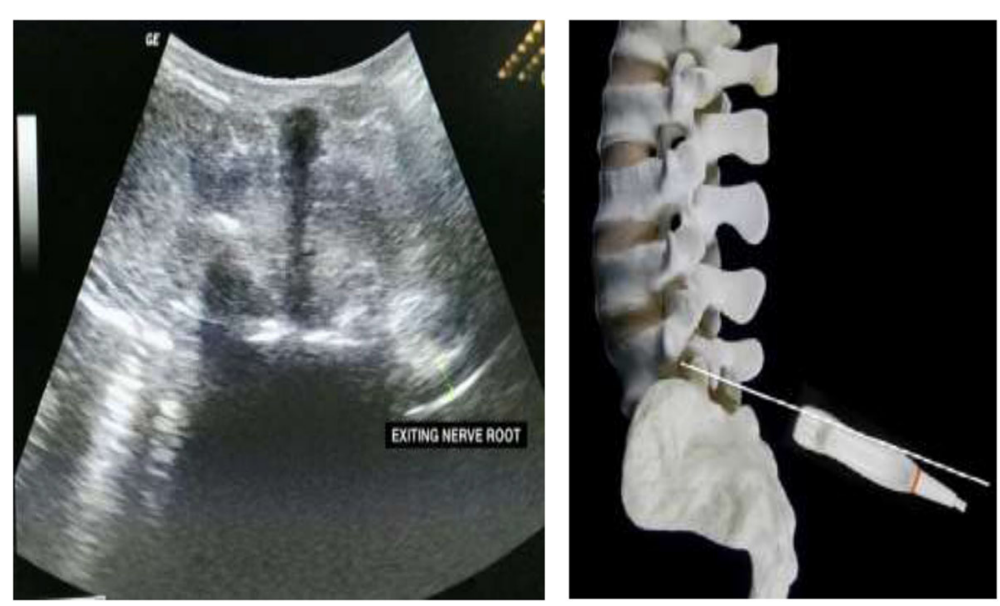

Fig. 2 a Axial sonogram of cadaveric lumbar vertebra shows lower edge of lamina and existing nerve root. b Cadaveric tilt 


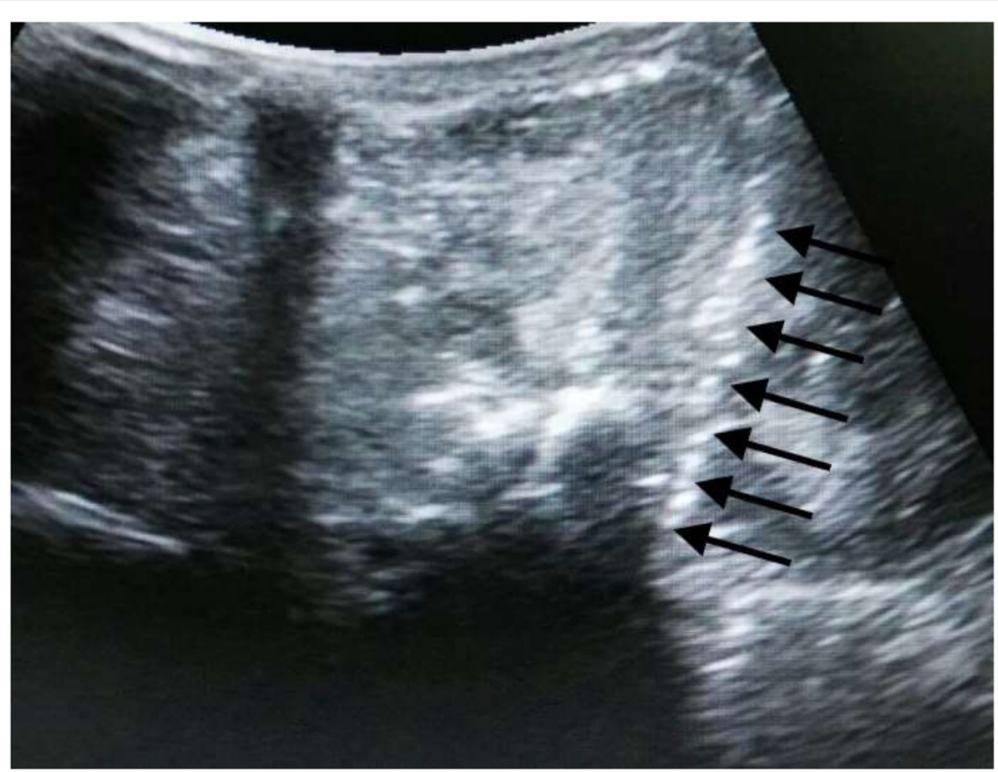

Fig. 3 Axial sonogram of lumbar vertebra shows entire length of needle tip medial and anterior to the inferior articular process

During needle progression, each patient was asked to be alert and to report immediately if they experienced any shooting pain or tingling in the leg that denoted touching nerve root. If this scenario happened the needle withdrawn $2-3 \mathrm{~mm}$ and redirected up and pain checked again.

Sensory stimulation of the DRG of the desired nerve root was tested by eliciting paresthesia in the dermatomal distribution of the affected nerve using an RF probe of neurotherm NT 2000 machine at $50 \mathrm{~Hz}$ and $0.4-0.6$

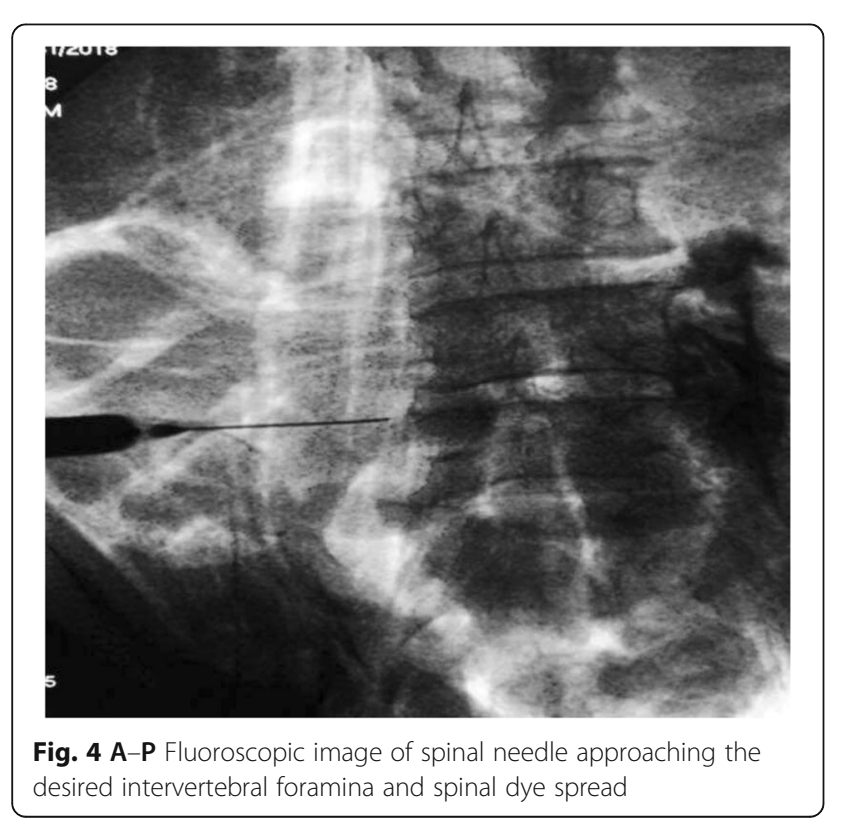

V. Fine adjustment of the PRF probe position might be done at this point. Motor stimulation was tested at $2 \mathrm{~Hz}$ and at voltage double that of the sensory threshold but at least at $1 \mathrm{~V}$.

Once satisfactory positive sensory stimulation, negative motor stimulation, and negative aspiration for blood or CSF were obtained; a lateral FL image was taken to confirm the cannula placement at the desired foraminal level. One milliliter of iohexol (Omnipaque) was injected under FL imaging to exclude intravascular injection and

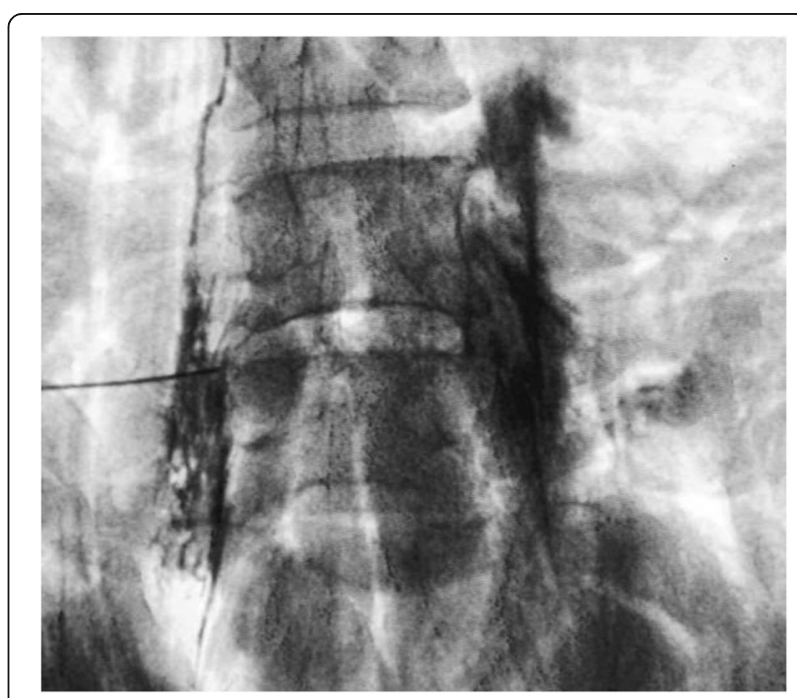

Fig. 5 A-P Fluoroscopic image of dye spread, the arrow points to the dural sleeve spread of dye, and the circle is overlying the intramuscular injection 


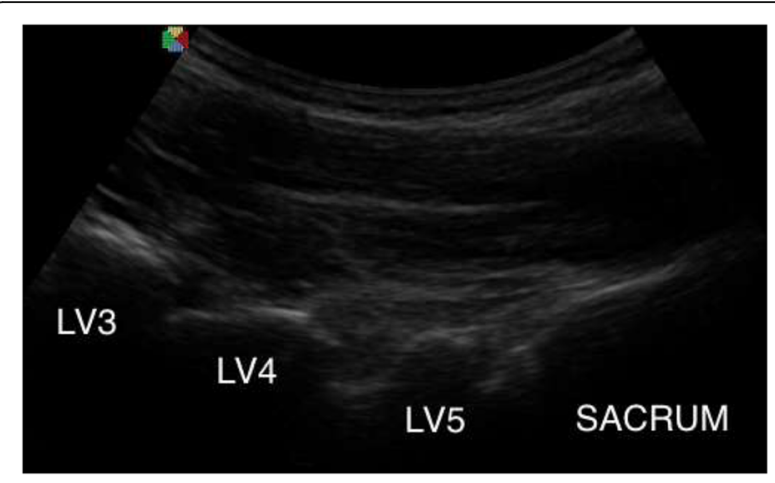

Fig. 6 Parasagittal oblique sonogram of the sacrum and laminae of lambar vertebrae

show distribution of the dye around nerve root. PRF wave was applied reaching $42^{\circ} \mathrm{C}$ and a pulse width of 20 ms for 4 min.

After the procedure, the patient was transferred to the recovery room for monitoring, observation, and management of any side effects. Any complication was reported and managed accordingly.

\section{Measurements}

1. The accuracy and time consumed during an ultrasound-guided DRG modulation

(a) The accuracy of pulsed radiofrequency cannula placement in a specific lumbar intervertebral space, adjacent to DRG guided with ultrasound and verified by fluoroscopy, was divided into 3 grades:

1 Grade II. Correct vertebral level, right cannula position in the intervertebral

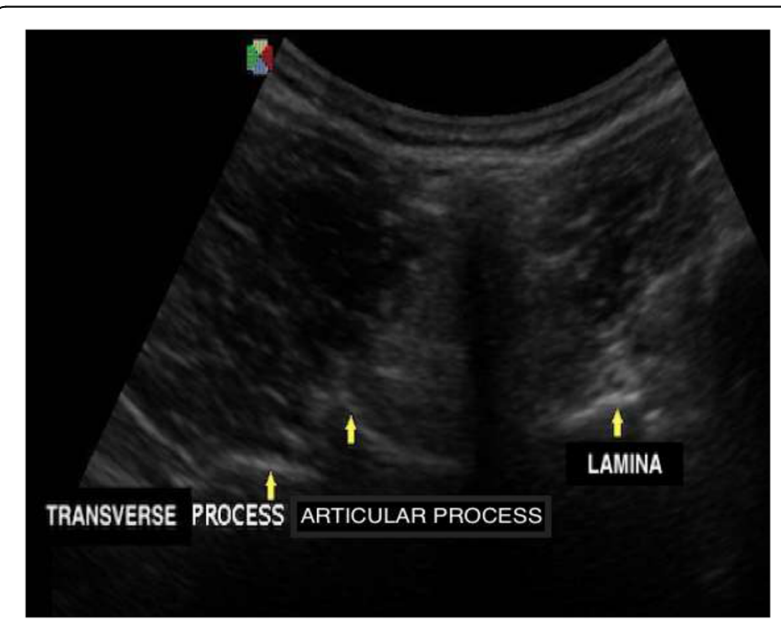

Fig. 7 The transverse axial sonogram of lumbar vertebra shows the spinous process, lamina, articular process, and transverse process

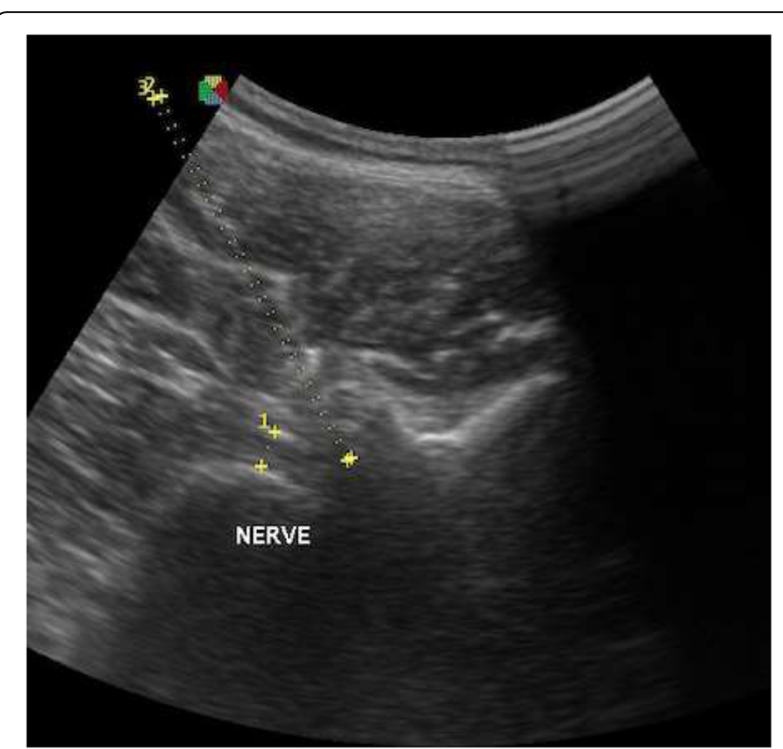

Fig. 8 Transverse axial oblique sonogram of lumbar vertebra shows nerve root and needle direction (yellow dots) towards DRG in front and medial to lateral border of inferior articular process

foramen, and positive sensory stimulation from a single entry trial.

2 Grade I. Correct vertebral level, right cannula position in the intervertebral foramen, and positive sensory stimulation with multiple trials; $\mathrm{Ia}=$ three trials, and $\mathrm{Ib}$ $=$ two trials.

3 Grade 0 . Incorrect vertebral level

(b) The procedure time consumed was measured in minutes.

1 Time needed for counting to the desired vertebral level.

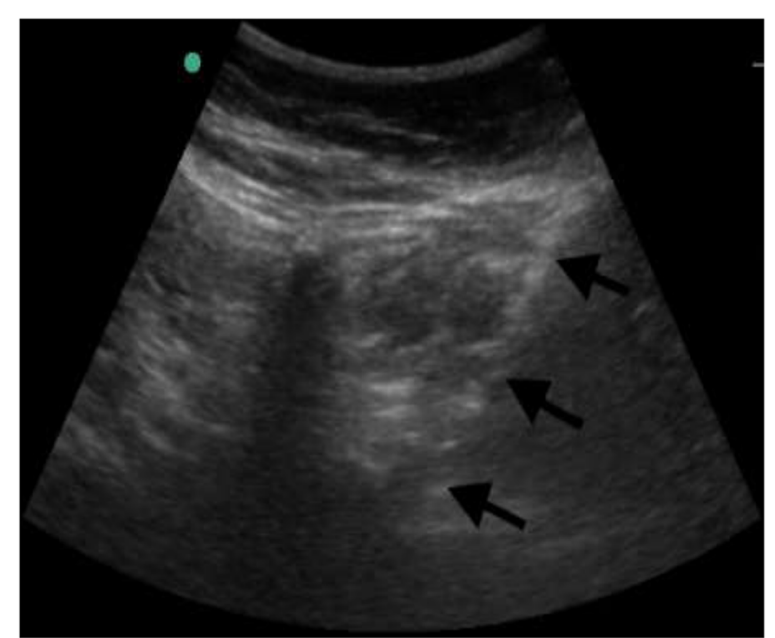

Fig. 9 Transverse axial sonogram of lumbar vertebra shows the entire path of the needle and needle tip anterior and medial to lateral border of articular process 


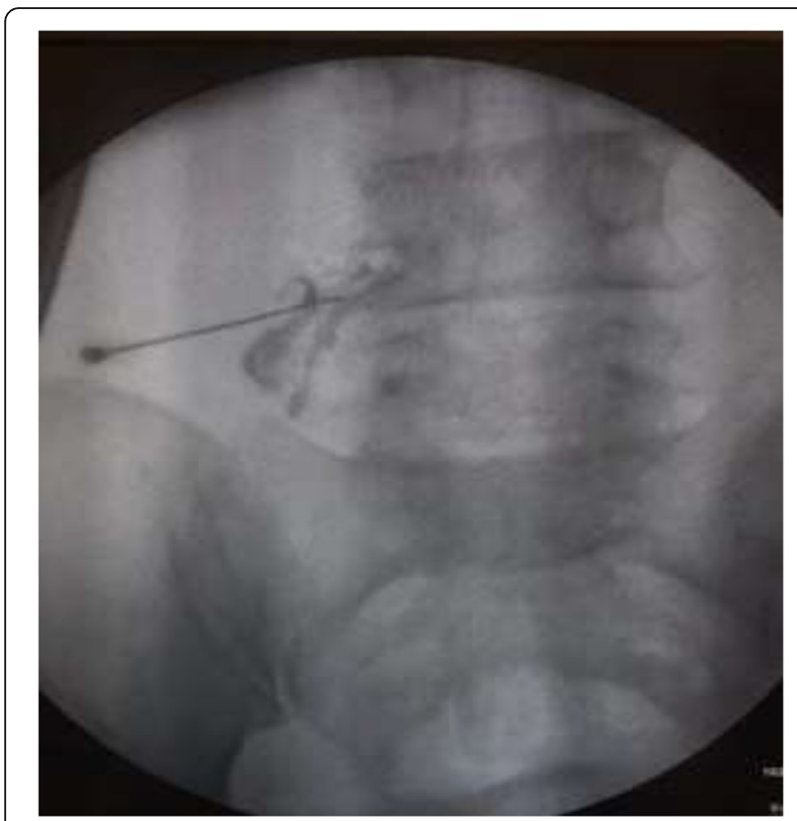

Fig. 10 A-P Fluoroscope verification of dye spread around nerve root

2 Time needed for visualization of the sonoanatomy of the vertebra.

3 Time needed to direct the cannula into intervertebral foramina, adjust the PRF cannula towards the DRG and stimulation and modulation of DRG.

2. Patients' radicular pain was measured at 2 weeks, 1 month, and 3 months post-intervention and compared to pre-intervention values using visual analogue scale of pain which is a $0-10 \mathrm{~cm}$ scale where 0 means no pain and 10 is the worst imaginable pain.

3. Patients' functionality was measured at 2 weeks, 1 month, and 3 months post-intervention and compared to pre-intervention values using Oswestry Low Back Pain Disability Questionnaire which is a ten-item scale; each item is rated from 0 to 5 (minimum to maximum impairment).On completing the test, the collected points are added, divided by 50 , and multiplied by 100 to obtain the percentage of disability or index. The higher the ODI index, the greater the disability (0 to $20 \%$, minimal disability; 21-40\%, moderate disability; $41-60 \%$, severe disability; $41-60 \%$, severe disability; $81-100 \%$, bed-bound).

4. Analgesic consumption reduction was calculated at 1 month post-intervention.

(a) If patient reported $\geq 75$ pain relief at the first meeting post-intervention, the analgesics reduced gradually through the next 2 weeks by $100 \%$. (b) If the reported pain relieved $\geq 50 \%$, the analgesics reduced gradually within 2 weeks by $50 \%$.

(c) If the reported pain relieved less than $50 \%$, the analgesics continued without dose reduction.

\section{Statistical methodology}

Data were collected and entered to the computer using SPSS (Statistical Package for Social Science) program for statistical analysis (ver. 21) [15]. Data were described using minimum, maximum, mean, standard deviation, and $95 \% \mathrm{CI}$ of the mean [16]. Comparisons were carried out between more than two independent normally distributed subgroups using one-way analysis of variance (ANOVA) test [17]. Post hoc multiple comparisons [18] was carried out using Games-Howell test [19]. Repeated measures analysis of variance was used [20]. Pair-wise comparison was done with Bonferroni correction. Diagnostic test evaluation was carried out using MedCalc Software version 14 [21]. An alpha level was set to 5\% with a significance level of $95 \%$ ( $p$ value $=0.05$ ).

\section{Results}

The study included one cadaver and 32 patients. Five lumbar trans-foraminal injections (L1/L2 through L5/S1) were performed bilaterally on whole preserved cadaver at anatomy laboratory with 8 correct injections out of 10. The spread of the injected dye was noticed in spinal space cephalic and bilateral.

Two injections were inadvertently done in paravertebral muscle and space, respectively. The incorrect injection was as a result of improper needle angulation into intervertebral foramen.

Thirty-two patients with CRP were included in the current study, 30 patients received unilateral interventions and 2 patients received L4 and L5 bilateral interventions with a total of 34 interventional procedures.

The age of the included 32 patients ranged from 30 to 60 years with a mean of $45.00 \pm 8.784$ years $(95 \%$ CI of the mean $41.83-48.17$ years). They were 15 (46.9\%) males and $17(53.1 \%)$ females. Eighteen interventions $(52.9 \%)$ were at right side, and $16(47.1 \%)$ interventions were at left side. The majority of interventions were at LV5 and LV4 DRG, 15 (44.1\%) for each level. Four interventions $(11.8 \%)$ were performed at LV3 DRG.

The overall accuracy of US-guided FL-verified DRGpulsed radiofrequency modulation was 91.18 (91.18 sensitivity) (95\% CI 76.32-98.14\%). Thirty-one US-guided RF cannulas insertions out of 34 (91.2\%) were successfully placed in a preselected lumbar intervertebral space, adjacent to DRG. Fifteen cannulas out of 34 (44.1\%) were successfully inserted from the first trial, 11 insertions (32.4\%) were from the second trial, and 5 (14.7\%) from more than 2 trials. Three $(8.8 \%)$ cannulas were 
placed in incorrect vertebral level (inaccurate placement, Fig. 11).

- Grade II. Correct vertebral level, right cannula position in the desired intervertebral foramen, and positive sensory stimulation from a single entry trial - Grade I. Correct vertebral level, right cannula position in the desired intervertebral foramen, and positive sensory stimulation with multiple trials; Ia = three trials, and $\mathrm{Ib}=$ two trials

- Grade 0. Incorrect vertebral level

The time taken for successful first trial RF cannula placement under US guidance ranged between 15 and 30 min with a mean of $17.73 \pm 1.51 \mathrm{~min}$ ( $95 \% \mathrm{CI}$ of the mean $19.17-21.86 \mathrm{~min})$. It was significantly shorter than the time consumed for more than one trial $(F=24.273$, $p=0.000)$. There was no significant difference in the time consumed between 2 and 3 trials.

The VAS of radicular pain ranged between 5 and 8 pre-intervention. It decreased significantly at 2 weeks, 4 weeks, and 3 months in comparison to the pre-operative intervention VAS value ( $p=0.000$, Fig. 12).

Patients reported severe to moderate disability index at pre-intervention time with a mean $=41.44 \pm 14.33$; this index decreased significantly at post-intervention time with mean of $12.19 \pm 10.40$ at 2 weeks, $11.25 \pm 9.46$ at 4 weeks, and $10.50 \pm 8.96$ at 3 months similar to VAS (Fig. 13).

Regarding analgesic consumption reduction, complete cessation was achieved in $53.1 \%$ of patients, decreased to half dose in $40.6 \%$ of patients, and no reduction in analgesic consumption in only $6.25 \%$ of patients, with an average of $73.44 \pm 31.07 \%$ in all patients.

The complications were minimal after intervention; 5 patients out of the 32 reported brief tenderness at site of injections that continued for 3 to 7 days. Ten patients out of the 32 patients experienced transient sensory changes in the ipsilateral leg which resolved in 1 week.

\section{Discussion}

The current study assumed that pulsed radiofrequency DRG modulation for radicular pain relief could be performed under US guidance. The US-guided lumbar pain interventions were thought to be complicated techniques; the high acoustic impedance of bone hides the underline structures and interrupts needle visualization. But frequent reviewing of sonoanatomy of lumbar region using different probe planes made better sonographic guidance for spinal interventions [12].

The present study showed that the overall incidence of accuracy of US-guided RF cannula insertion in a preselected lumbar intervertebral foraminal space adjacent to DRG was $91.18 \%$ with minimal risk.

The US-curved probe was used in parasagittal oblique plane for cephalic counting to desired vertebral level and in transverse plane, below the level of transverse process for visualization of the details of vertebra and existing nerve root and directing RF cannula in plane from lateral to median towards lateral border of inferior articular process. Up till now, there is no standardized technique for lumbar trans-foraminal injection.

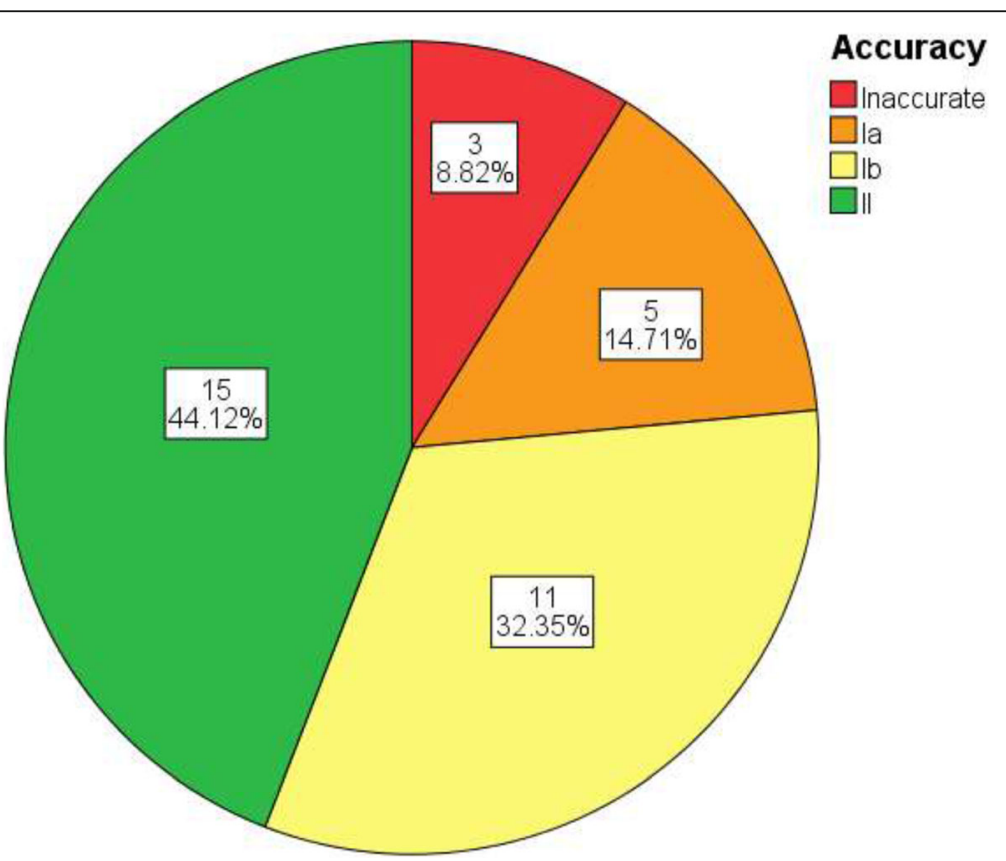

Fig. 11 Pie chart showing the accuracy of US-guided fluoroscopic-verified dorsal root ganglia pulsed radiofrequency cannula placement 


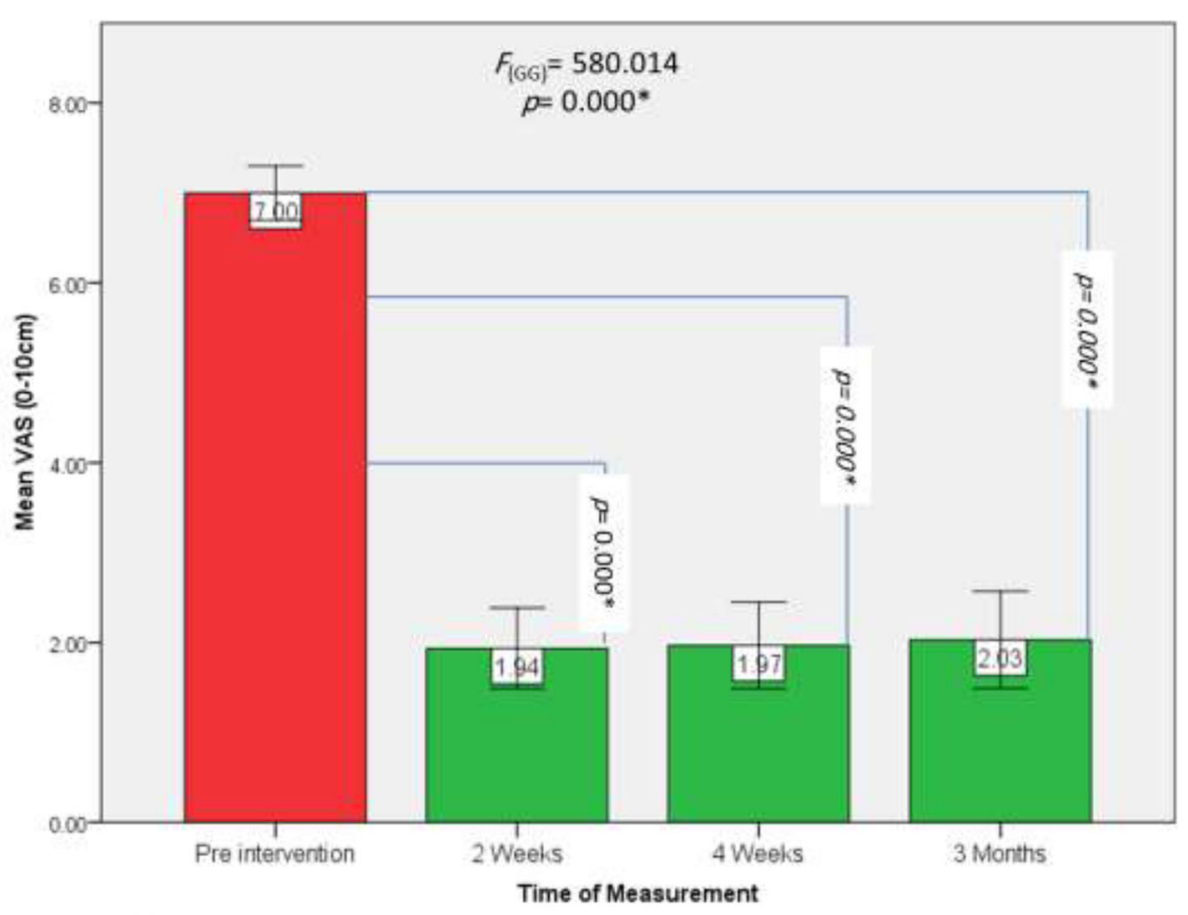

Error Bars: $95 \% \mathrm{Cl}$

Fig. 12 Bar chart with 95\% Cl error bar, comparison between the pre- and post-intervention mean visual analogue scale (VAS) level

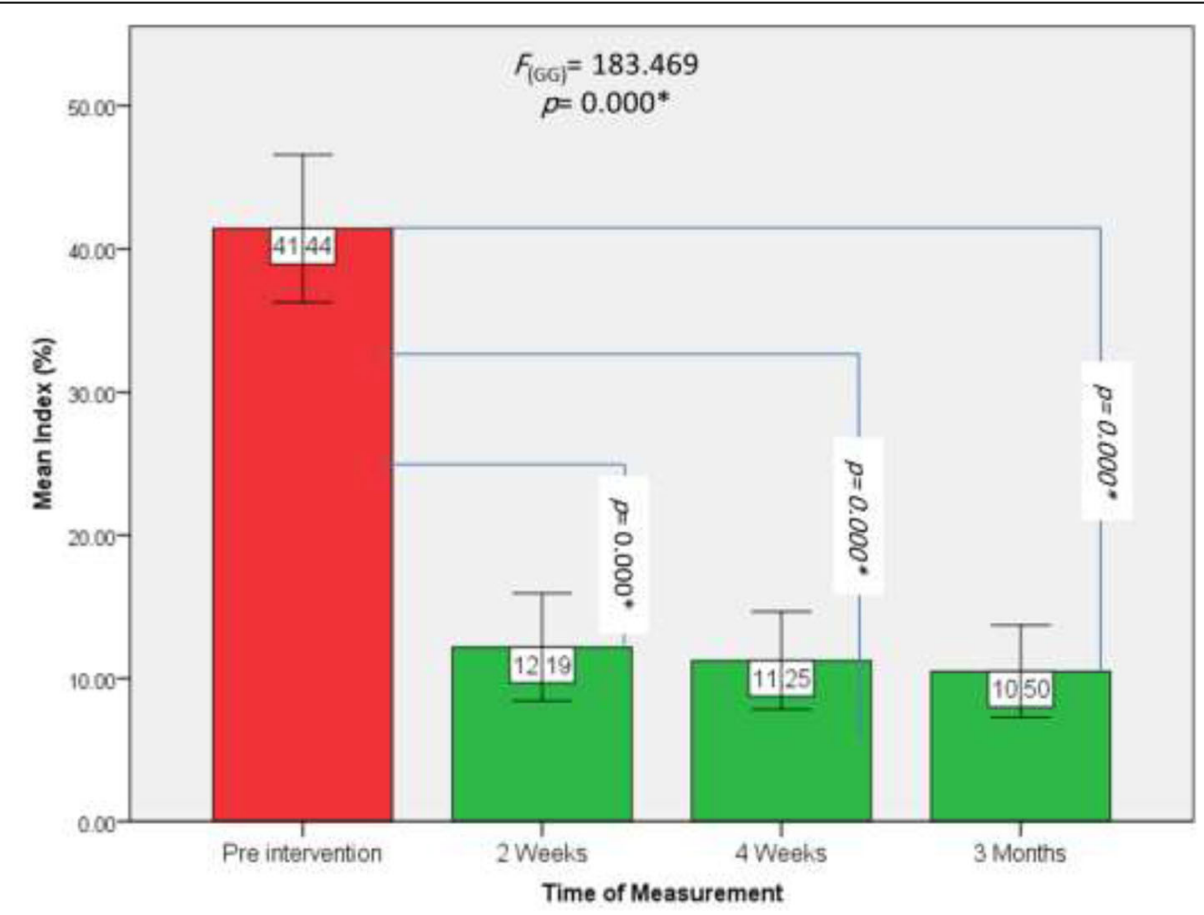

Enor Bars: $95 \% \mathrm{Cl}$

Fig. 13 Bar chart with 95\% Cl error bar, comparison between pre- and post-intervention Oswestry disability index 
Wan et al. reported 95\% success of US-guided lumbar peri-radicular injections for unilateral radicular pain. They compared the axial approach with the parasagittal approach. A curvilinear US probe was placed transversely over the spinous process in transverse axial scan to visualize the spinous process, lamina, facet joints, and transverse process. In-plane spinal needle was directed to the lateral edge of the lamina or medial to superior articular process. The same probe was used in parasagittal plane to show edges of facet joints and out of plane spinal needle was inserted at $70^{\circ}$ over the skin to place the needle tip between the adjacent facet joints. CT was used to confirm needle tip position. None of the patients reported any intervention-related side effects [22].

Yang et al. used US as guidance for trans-foraminal epidural steroid injection for lumbar radicular pain relief with $85 \%$ accuracy. They used a linear probe in midline sagittal plane for cephalic counting to the target lumbar level and in axial view to show details of vertebra and guide spinal needle in plane towards lateral edge of lamina. Then, they rotated US probe $90^{\circ}$ to be placed parasagittal and advanced the tip of the needle between the adjacent facet joints. After that, the needle tip was slightly moved backward and slipped beside the lamina, and advanced a little deeper until the loss of resistance test was positive. The final tip of needle position was confirmed by FL [23].

Chumnanvej et al. reported $80.95 \%$ accuracy of USguided lumbar peri-radicular corticosteroid and local anesthetic injection for radicular pain confirmed by fluoroscopy. A curved US probe was used in parasagittal planeee to count to desired lumbar level. Then, axial plane was used to guide tip of spinal needle lateral to facet joint. L4-5 facet was used as a land mark for L4 nerve root injection and L5-S1 for L5 root. The skin entry point of the needle insertion is about $10 \mathrm{~cm}$ from midline or, at least, more lateral to the tip of transverse process. For the L5-S1 level, the spinal needle was placed just lateral to the ultrasound probe as ala of sacrum interfered with site of needle entry. Subsequent fluoroscopy was checked in AP and lateral view for needle tip confirmation [24].

In the present study, the grade II accuracy which was successful first trial placement of pulsed radiofrequency cannula in the desired lumbar intervertebral foramine, adjacent to DRG, was recorded in $44.1 \%$ of interventions; multiple trials were needed in $47.1 \%$ of interventions while incorrect placement was encountered in $8.8 \%$ of interventions.

Higher accurate incidence of tip needle position was recorded in Chumnanvej et al.'s research. They studied accuracy of needle tip localization by ultrasound guidance close to 78 lumbar nerve roots. Forty-nine nerve roots out of $78(62.8 \%)$ belonged to category II, which was defined as optimal needle tip position, at both the desired intervertebral level and exact right position. Twenty-six roots (33.3\%) belonged to category 1 where needle tip was at desired intervertebral level but not in the exact position subsequently confirmed by fluoroscopy. Category 1 in their study might need more than a trial for proper placement. Three roots (3.8\%) were related to category 0 with incorrect vertebral level. The discrepancy of accurate incidence between their study and ours could be attributed to different target of needle position [24].

In the present study, the time needed for successful first US-guided DRG PRF trial was $17.7 \pm 1.5 \mathrm{~min}$. Longer time was taken to perform second and third trials $(22.18 \pm 2.3$ and $25.2 \pm 3.9 \mathrm{~min}$, respectively). Relatively similar results was published in Sahu et al.'s study; they reported $16.2 \pm 1.5 \mathrm{~min}$ as an average time for one attempt TFESI and $25.1 \pm 1 \mathrm{~min}$ for two-attempts [25].

In the current study, the VAS of radicular pain decreased significantly at 2 week, 4 week, and after 3 months compared to the pre-intervention value. The reduced pain severity reflected positively on analgesic consumption that showed complete cessation of analgesic consumption in $53.1 \%$ of patients, decrease to half dose in $40.6 \%$ of patients, and no reduction in analgesic consumption in only $6.25 \%$ of patients.

In agreement with the present study, Khalifa and Saadalla reported that lumber DRG PRF intervention reduced radicular pain due to disc herniation up to 3 months. The author compared lumber DRG PRF with lumbar trans-foraminal epidural steroid injection to detect which was more effective. They concluded that lumber DRG PRF was as effective as lumbar transforaminal epidural steroid injection but with more prolonged duration [26].

Patients reported severe to moderate ODI at preintervention time with mean value of $41.44 \pm 14.33$. After intervention, patients' functionality improved as a result of decreased ODI at 2 and 4 weeks with mean values of $12.19 \pm 10.40$ and $11.25 \pm 9.46$, respectively.

The reduction of post-intervention analgesic consumption and ODI were reported in Trinidad et al.'s research who studied the effect of PRF for 25 patients with radicular pain. Sixty-eight percent of studied patients reduced their consumption of analgesics with respect to the baseline levels and almost 35\% achieved a complete cessation of medication. ODI score was $51.08 \%$ at pre-intervention time and reduced to $15.28 \% 1$ month after radiofrequency treatment which reflected significant improvement of patients activities [27].

\section{Risks and complications}

Overall, percutaneous US-guided pulsed RF application of lumbar dorsal toot ganglion is a safe procedure and is 
associated with only minimal risk for bleeding and infection. There have been reported cases of transient motor and sensory deficits in the ipsilateral leg related to the transient effect of the injected local anesthetic.

\section{Conclusion}

US-guided trans-foraminal PRF DRG modulation using curved US probe in axial plane below the level of transverse process of selected vertebra with cannula insertion from lateral edge of probe towards inferior articular process and just medial and anterior to it, subsequently confirmed by FL and electrical sensory stimulation is accurate, effective, and safe technique for lumbar radicular pain management. Most of our patients were overweight; however, we did not do the correlation between the outcome and body mass index of patients statistically, so we do recommend its addition in further research.

\section{Abbreviations}

AP: Articular process; CRP: Chronic radicular pain; DRG: Dorsal root ganglion; FL: Fluoroscopy; PRF: Pulsed radiofrequency; US: Ultrasound

\section{Acknowledgements}

Not applicable.

\section{Authors' contributions}

SO: Design of the study, data collection, US and fluoroscopy image interpretation, statistical analysis, and manuscript writing. SEH: Design of the study, fluoroscopy image interpretation, and manuscript editing. SAE: Design of the study, initial and continuous training on the technique, US and fluoroscopy image interpretation, and manuscript editing. MISR: Design of the study, fluoroscopy image interpretation, and manuscript editing. MMS: Patient referral and manuscript editing. NME: Reviewing the anatomy portion of the study and aiding in cadaveric work. OSE: Design of the study and manuscript editing. SO, SAE, SEH, MISR, MMS, NME, and OSE: All authors read and approved the final manuscript.

\section{Funding}

No sources of funding.

\section{Availability of data and materials}

The datasets used and/or analyzed during the current study are available from the corresponding author on reasonable request.

\section{Ethics approval and consent to participate}

This study was approved by the Research Ethics Committee of the Faculty of Medicine at Alexandria University in Egypt on March 2018 (reference number is 0201080). All patients included in this study gave written informed consent to participate in this research.

\section{Consent for publication}

All patients included in this research gave written informed consent to publish the data contained within this study.

\section{Competing interests}

The authors declare that they have no competing interests.

\section{Author details}

'Department of Diagnostic and Interventional Radiology, Faculty of Medicine, Alexandria University, Alexandria, Egypt. ${ }^{2}$ Department of Anaesthesia and Pain Management, Medical Research Institute, Alexandria University, Alexandria, Egypt. ${ }^{3}$ Department of Physical Medicine, Rheumatology and Rehabilitation, Faculty of Medicine, Alexandria University, Alexandria, Egypt. ${ }^{4}$ Department of Anatomy, Faculty of Medicine, Alexandria University, Alexandria, Egypt.
Received: 23 October 2020 Accepted: 13 January 2021

Published online: 29 January 2021

\section{References}

1. Alexanderm CE, Varacallo M (2019) Lumbosacral radiculopathy. StatPearls Publishing, Treasure Island

2. Espeland A, Dalen N (2019) Disc and nerve root findings on lumbar MR with straightened $v$ s flexed hips and knees-pilot study. Br J Radiol 92(1096): 20180851

3. Yang S, Kim W, Kong HH, Do KH, Choi KH (2020) Epidural steroid injection versus conservative treatment for patients with lumbosacral radicular pain: a meta-analysis of randomized controlled trials. Medicine 99(30):e21283

4. Van Boxem K, Cheng J, Patijn J, van Kleef M, Lataster A, Mekhail N et al (2010) 11. Lumbosacral radicular pain. Pain Pract 10(4):339-358

5. Hunter CW, Sayed D, Lubenow T, Davis T, Carlson J, Rowe J et al (2019) DRG FOCUS: a multicenter study evaluating dorsal root ganglion stimulation and predictors for trial success. Neuromodulation 22(1):61-79

6. Shen J, Wang HY, Chen JY, Liang BL (2006) Morphologic analysis of normal human lumbar dorsal root ganglion by 3D MR imaging. AJNR Am J Neuroradiol 27(10):2098-2103

7. Tortora F, Negro A, Russo C, Cirillo S, Caranci F (2020) Chronic intractable lumbosacral radicular pain, is there a remedy? Pulsed radiofrequency treatment and volumetric modifications of the lumbar dorsal root ganglia. Radiol Med. https://doi.org/10.1007/s11547-11020-01212-z

8. Deshmukh S, Youngner J, Garg A (2020) Fluoroscopy-guided spine injections: establishing a successful service in your radiology department or practice. Skelet Radiol 49(3):475-479

9. Hashemi M, Dadkhah P, Taheri M, Haji Seyed Abootorabi SM, Naderi-Nabi B (2019) Ultrasound-guided lumbar transforaminal epidural injections; a single center fluoroscopic validation study. Bull Emerg Trauma 7(3):251-255

10. Yoon SH, O'Brien SL, Tran M (2013) Ultrasound guided spine injections: advancement over fluoroscopic guidance? Curr Phys Med Rehabil Rep 1(2): $104-113$

11. Liao JJ (2010) Sample size calculation for an agreement study. Pharm Stat 9(2):125-132

12. Provenzano DA, Narouze S (2013) Sonographically guided lumbar spine procedures. J Med Ultrasound 32(7):1109-1116

13. Gofeld M, Bristow SJ, Chiu SC, McQueen CK, Bollag L (2012) Ultrasoundguided lumbar transforaminal injections: feasibility and validation study. Spine 37(9):808-812

14. Galiano K, Obwegeser AA, Bodner G, Freund M, Maurer H, Kamelger FS et al (2005) Real-time sonographic imaging for periradicular injections in the lumbar spine: a sonographic anatomic study of a new technique. J Ultrasound Med 24(1):33-38

15. IBM Corp (2012) IBM SPSS Statistics for Windows, Version 21.0. IBM Corp., Armonk

16. Snecdecor GW, Cochran WG (1991) Statistical methods. Wiley, New Jersey

17. Montgomery D (2001) The analysis of variance. In: Montgomery D (ed) Design and analysis of experiments. Wiley, Hoboken

18. Lowry R One way ANOVA-independent samples. Vassar. edu. Retrieved on 4 Dec 2008 Retrieved on 4 Dec 2008

19. Ruxton GD, Beauchamp G (2008) Time for some a priori thinking about post hoc testing. Behav Ecol 19(3):690-693

20. Gueorguieva R, Krystal JH (2004) Move over ANOVA: progress in analyzing repeated-measures data and its reflection in papers published in the Archives of General Psychiatry. Arch Gen Psychiatry 61(3):310-317

21. DeLong ER, DeLong DM, Clarke-Pearson DL (1988) Comparing the areas under two or more correlated receiver operating characteristic curves: a nonparametric approach. Biometrics 44(3):837-845

22. Wan Q, Wu S, Li X, Lin C, Ke S, Liu C et al (2017) Ultrasonography-guided lumbar periradicular injections for unilateral radicular pain. Biomed Res Int 2017:8784149

23. Yang G, Liu J, Ma L, Cai Z, Meng C, Qi S et al (2016) Ultrasound-guided versus fluoroscopy-controlled lumbar transforaminal epidural injections: a prospective randomized clinical trial. Clin J Pain 32(2):103-108

24. Chumnanvej S, Wetchagama N, Kounsongtham V (2011) Accuracy of needle-tip localization by ultrasound guidance lumbar selective nerve root block: a prospective clinical study of 78 lumbar nerve roots block. J Med Assoc Thail 94(12):1451-1456 
25. Sharma A, Kothari K, Wani P, Patel C, Parampill R (2016) Ultrasound-guided fluoroscopic-verified lumbar transforaminal epidural injection: a clinical evaluation of technique. Indian J Pain 30:158

26. Khalifa O, Saadalla A (2017) Steroids versus pulsed radiofrequency in treatment of radicular pain due to lumbar disc prolapse: a randomized clinical trial. Res Opin Anesth Intensive Care 4(4):184-187

27. Trinidad JM, Carnota Al, Failde I, Torres LM (2015) Radiofrequency for the treatment of lumbar radicular pain: impact on surgical indications. Pain Res Treat 2015:392856

\section{Publisher's Note}

Springer Nature remains neutral with regard to jurisdictional claims in published maps and institutional affiliations.

\section{Submit your manuscript to a SpringerOpen ${ }^{\mathcal{O}}$ journal and benefit from:}

- Convenient online submission

- Rigorous peer review

- Open access: articles freely available online

High visibility within the field

- Retaining the copyright to your article

Submit your next manuscript at $\boldsymbol{\nabla}$ springeropen.com 